\title{
Griffiths-McCoy Singularities in the Random Transverse-Field Ising Spin Chain
}

\author{
Ferenc Iglói ${ }^{1,2}$, Róbert Juhász ${ }^{2,1}$ and Heiko Rieger ${ }^{3,4}$ \\ 1 Research Institute for Solid State Physics and Optics, H-1525 Budapest, P.O.Box 49, Hungary \\ ${ }^{2}$ Institute for Theoretical Physics, Szeged University, H-6720 Szeged, Hungary \\ ${ }^{3}$ Institut für Theoretische Physik, Universität zu Köln, 50923 Köln, Germany \\ ${ }^{4}$ NIC c/o Forschungszentrum Jülich, 52425 Jülich, Germany
}

(November 11, 1998)

\begin{abstract}
We consider the paramagnetic phase of the random transverse-field Ising spin chain and study the dynamical properties by numerical methods and scaling considerations. We extend our previous work [Phys. Rev. B 57, 11404 (1998)] to new quantities, such as the non-linear susceptibility, higher excitations and the energy-density autocorrelation function. We show that in the Griffiths phase all the above quantities exhibit power-law singularities and the corresponding critical exponents, which vary with the distance from the critical point, can be related to the dynamical exponent $z$, the latter being the positive root of $\left[(J / h)^{1 / z}\right]_{\text {av }}=1$. Particularly, whereas the average spin autocorrelation function in imaginary time decays as $[G]_{\text {av }}(\tau) \sim \tau^{-1 / z}$, the average energy-density autocorrelations decay with another exponent as $\left[G^{e}\right]_{\text {av }}(\tau) \sim \tau^{-2-1 / z}$.
\end{abstract}

\section{INTRODUCTION}

Quantum phase transitions occur at zero temperature by varying a parameter of the Hamiltonian, e.g. the strength of a transverse field. Quenched, i.e. time independent disorder has generally a profound effect on the properties of the quantum system not only at the critical point, but also in a whole region, which extends in both sides of the critical point. In this so called Griffiths phase the dynamical properties of the random quantum systems are exceptional: for example the (imaginary) time dependent average spin-spin correlations decay algebraically [1]

$$
[G]_{\mathrm{av}}(\tau) \sim \tau^{-1 / z(\delta)}
$$

where the dynamical exponent $z(\delta)$ is a continuous function of the quantum control parameter, $\delta$. From here on we use $[\ldots]_{\text {av }}$ to denote averaging over quenched disorder. The physical origin of this type of singular behavior, as was pointed out by Griffiths [2] for classical systems, is the existence of clusters in the random system, which are more strongly coupled than the average. The spins of such clusters, being locally in the "ordered phase", behave coherently as a giant spin and the corresponding relaxation time is very large. Thus in an infinite system there is no (finite) time-scale and, as a consequence, the autocorrelations decay algebraically, as in (1.1) [3].

Several physical quantities, which involve an integral of the autocorrelation function, (e.g. the static susceptibility,) are singular not only at the critical point but also in a finite region of the paramagnetic phase. This phenomenon has been first noticed by McCoy in a two-dimensional classical model with correlated disorder, (equivalent to a one-dimensional random quantum model) [4], therefore we call the Griffiths singularities in quantum systems Griffiths-McCoy singularities.
Many of the theoretical studies on random quantum systems are related to random quantum ferromagnets [5] and quantum spin glasses [6], which have also experimental realizations [7]. In higher $(d=2$ and $d=3)$ dimensions one generally studies the distribution of the (linear and non-linear) susceptibilities, the asymptotic behavior of those can be related to the dynamical exponent, $z(\delta)$, by scaling considerations. According to numerical studies - in agreement with these phenomenological theories $z(\delta)$ is found continuous function of the quantum control parameter $\delta$, which appear to have a finite limiting value at the critical point, $\delta=0$, of spin glasses $\|6\|$, whereas it is diverging for random ferromagnets 5 .

Many features of Griffiths-McCoy singularities can already be seen in one-dimensional systems, where many exact and conjectured results exist. In this paper we consider the prototype of random quantum systems the random transverse-field Ising model (RTIM) in one dimension, defined by the Hamiltonian:

$$
H=-\sum_{l} J_{l} \sigma_{l}^{x} \sigma_{l+1}^{x}-\sum_{l} h_{l} \sigma_{l}^{z} .
$$

Here the $\sigma_{l}^{x}, \sigma_{l}^{z}$ are Pauli matrices at site $l$ and the $J_{l}$ exchange couplings and the $h_{l}$ transverse-fields are random variables with distributions $\pi(J)$ and $\rho(h)$, respectively. Note that in one dimension all the couplings and fields can be taken positive through a gauge transformation. The model in $(1.2)$ is in the ferromagnetic (paramagnetic) phase if the couplings in average are stronger (weaker) than the transverse fields. As a convenient quantum control-parameter one can define:

$$
\delta=\frac{[\ln h]_{\mathrm{av}}-[\ln J]_{\mathrm{av}}}{\operatorname{var}[\ln \mathrm{h}]+\operatorname{var}[\ln \mathrm{J}]},
$$

and at the critical point $\delta=0$.

The Hamiltonian in eq(1.2) is closely related to the transfer matrix of a classical two-dimensional layered 
Ising model, which was first introduced and partially solved by McCoy and $\mathrm{Wu}$ 8]. Later the critical properties of the quantum model was studied by Shankar and Murthy [9], and in great detail by Fisher [10]. Through a renormalization group (RG) transformation Fisher has obtained many new results on static quantities and equal time correlations, which are claimed to be exact for large scales, i.e. in the vicinity of the critical point. Many of Fisher's results have been checked numerically 11] and in addition new results have been obtained about critical density profiles 12], time-dependent critical correlations [13] and various probability distributions and scaling functions [11,14. Later, using simple expressions about the surface magnetization and the energy gap several exact results have been derived by making use of a mathematical analogy with surviving random walks [14, see also [15].

In the Griffiths phase, where the RG results are restricted to the immediate vicinity of the critical point, i.e. as $\delta \rightarrow 0$, numerical investigations both on temperature dependent [16] (specific heat, susceptibility) and dynamical quantities (spin-spin autocorrelations, distribution of the energy gap and susceptibility) [14,11 have lead to the conclusion, that the behavior of all these quantities is a consequence of Griffiths-McCoy singularities and can be characterized by a single varying exponent $z(\delta)$ in (1.1). Very recently an analytical expression for $z(\delta)$ has been derived [17] by using an exact mapping [18] between the Hamiltonian in (1.2) and the Fokker-Planck operator of a random walk in a random environment. The dynamical exponent, which is given by the positive root of the equation:

$$
\left[\left(\frac{J}{h}\right)^{1 / z}\right]_{\mathrm{av}}=1,
$$

generally depends both on $\delta$ and on the distributions $\pi(J)$ and $\rho(J)$. However it becomes universal, i.e. distribution independent, in the vicinity of the critical point when $z(\delta) \approx 1 /(2 \delta),|\delta| \ll 1$, in accordance with the RG results [10]. The numerical results obtained about different singular quantities in the Griffiths phase are all in agreement with the analytical formula in (1.4) and the observed small deviations are attributed to finite-size corrections [16, 14].

The singular quantities studied so far in the Griffiths phase are all related to the scaling properties of the lowest energy gap, which explains the observation why a single varying exponent is sufficient to characterize the singularities of the different quantities. There are, however, other observables, which are expected to be singular too, but not connected directly to the first gap. For example one could consider the distribution of the second (or some higher) gap. By similar reasons as for the first gap these higher excitations are also expected to vanish in the thermodynamic limit and the corresponding probability distributions are described by new exponents for small values of the gaps. As another example we consider the connected transverse spin autocorrelation function $G_{l}^{e}(\tau)=\left\langle\left\langle\sigma_{l}^{z}(0) \sigma_{l}^{z}(\tau)\right\rangle\right\rangle$. In the two-dimensional classical version of (1.2), the McCoy-Wu model, this function corresponds to the energy-density correlation function in the direction, where the disorder is correlated. Therefore we adopt in the following this terminology and call $G_{l}^{e}(\tau)$ the energy-density autocorrelation function. Since the inverse time scale for these correlations is, as we shall see, determined by the second gap, one expects that also $\left[G^{e}\right]_{\mathrm{av}}(\tau)$ has an algebraic decay:

$$
\left[G^{e}\right]_{\mathrm{av}}(\tau) \sim \tau^{-\eta_{e}},
$$

with an exponent $\eta_{e}$. Finally one should mention the non-linear susceptibility whose distribution is expected to be described by a new varying exponent.

In this paper we extend previous numerical work and study the scaling behavior of the above mentioned singular quantities in the Griffiths phase. We present a phenomenological scaling theory and we confront its predictions by results of numerical calculations, based on the free-fermion representation of the Hamiltonian in (1.2). We show that the physical quantities we studied are characterized by power-law singularities with varying critical exponents, the value of those are connected to the dynamical exponent through scaling relations.

Throughout the paper we use two types of random distributions. In the symmetric binary distribution the couplings could take two values $\lambda>1$ and $1 / \lambda$ with the same probability, while the transverse-field is constant:

$$
\begin{array}{r}
\pi(J)=\frac{1}{2}\left(\delta(J-\lambda)+\delta\left(J-\lambda^{-1}\right)\right), \\
\rho(h)=\delta\left(h-h_{0}\right) .
\end{array}
$$

At the critical point $h_{0}=1$, whereas in the Griffiths phase, $1<h_{0}<\lambda$, the dynamical exponent from (1.4) is determined by the equation:

$$
h_{0}^{1 / z}=\cosh \left(\frac{\ln \lambda}{z}\right) .
$$

In the uniform distribution both the couplings and the fields have rectangular distributions:

$$
\begin{gathered}
\pi(J)= \begin{cases}1, & \text { for } 0<J<1 \\
0, & \text { otherwise }\end{cases} \\
\rho(h)= \begin{cases}h_{0}^{-1}, & \text { for } 0<h<h_{0} \\
0, & \text { otherwise }\end{cases}
\end{gathered}
$$

The critical point is also at $h_{0}=1$, whereas the dynamical exponent is given by the solution of the equation:

$$
z \ln \left(1-z^{-2}\right)=-\ln h_{0},
$$

where the Griffiths phase now extends to $1<h_{0}<\infty$.

The structure of the paper is the following. In Section 2. we present the free fermion description of various dynamical quantities. Phenomenological and scaling considerations are given in Section 3, the numerical results are presented in Section 4. Finally, we close the paper with a Discussion. 


\section{FREE FERMION DESCRIPTION OF DYNAMICAL QUANTITIES}

We consider the random transverse-field Ising model in eq(1.2) on a finite chain of length $L$ with free boundary conditions. The Hamiltonian in eq(1.2) is mapped through a Jordan-Wigner transformation and a following canonical transformation [19] into a free fermion model:

$$
H=\sum_{q=1}^{L} \epsilon_{q}\left(\eta_{q}^{+} \eta_{q}-\frac{1}{2}\right)
$$

in terms of the $\eta_{q}^{+}\left(\eta_{q}\right)$ fermion creation (annihilation) operators. The energy of modes, $\epsilon_{q}$, is obtained through the solution of an eigenvalue problem, which necessitates the diagonalization of a $2 L \times 2 L$ tridiagonal matrix with nonvanishing matrix-elements $T_{2 i-1,2 i}=T_{2 i, 2 i-1}=h_{i}, i=$ $1,2, \ldots, L$ and $T_{2 i, 2 i+1}=T_{2 i+1,2 i}=J_{i}, i=1,2, \ldots, L-1$, and denote the components of the eigenvectors $V_{q}$ as $V_{q}(2 i-1)=-\phi_{q}(i)$ and $V_{q}(2 i)=\psi_{q}(i), i=1,2, \ldots, L$, i.e.

$$
T=\left(\begin{array}{ccccccc}
0 & h_{1} & & & & & \\
h_{1} & 0 & J_{1} & & & & \\
0 & J_{1} & 0 & h_{2} & & & \\
& & h_{2} & 0 & \ddots & & \\
& & & \ddots & \ddots & J_{L-1} & \\
& & & & J_{L-1} & 0 & h_{L} \\
& & & & & h_{L} & 0
\end{array}\right) \quad, \quad V_{q}=\left(\begin{array}{c}
-\Phi_{q}(1) \\
\Psi_{q}(1) \\
-\Phi_{q}(2) \\
\vdots \\
\Psi_{q}(L-1) \\
-\Phi_{q}(L) \\
\Psi_{q}(L)
\end{array}\right)
$$

We consider only the $\epsilon_{q} \geq 0$ part of the spectrum [20].

The local susceptibility $\chi_{l}$ at site $l$ is defined through the local magnetization $m_{l}$ as:

$$
\chi_{l}=\lim _{H_{l} \rightarrow 0} \frac{\delta m_{l}}{\delta H_{l}},
$$

where $H_{l}$ is the strength of the local longitudinal field, which enters the Hamiltonian (1.2) via an additional term $H_{l} \sigma_{l}^{x} \cdot \chi_{l}$ can be expressed as:

$$
\chi_{l}=2 \sum_{i} \frac{\left|\left\langle i\left|\sigma_{l}^{x}\right| 0\right\rangle\right|^{2}}{E_{i}-E_{0}}
$$

where $|0\rangle$ and $|i\rangle$ denote the ground state and the $i$ th excited state of $H$ in (1.2) with energies $E_{0}$ and $E_{i}$, respectively. For boundary spins one has the simple expression:

$$
\chi_{1}=2 \sum_{q} \frac{\left|\phi_{q}(1)\right|^{2}}{\epsilon_{q}}
$$

Similarly, the local non-linear susceptibility is defined by:

$$
\chi_{l}^{\mathrm{nl}}=\lim _{H_{l} \rightarrow 0} \frac{\delta^{3} m_{l}}{\delta H_{l}^{3}},
$$

and can be expressed as:

$$
\chi_{l}^{\mathrm{nl}}=24\left\{\sum_{i, j, k}\left\langle 0\left|\sigma_{l}^{x}\right| i\right\rangle \frac{1}{E_{i}-E_{0}}\left\langle i\left|\sigma_{l}^{x}\right| j\right\rangle \frac{1}{E_{j}-E_{0}}\left\langle j\left|\sigma_{l}^{x}\right| k\right\rangle \frac{1}{E_{k}-E_{0}}\left\langle k\left|\sigma_{l}^{x}\right| 0\right\rangle+\sum_{i}\left(\frac{\left\langle i\left|\sigma_{l}^{x}\right| 0\right\rangle}{E_{i}-E_{0}}\right)^{2} \sum_{j} \frac{\left|\left\langle j\left|\sigma_{l}^{x}\right| 0\right\rangle\right|^{2}}{E_{j}-E_{0}}\right\} .
$$

It should be noted that it is not the first sum on the r.h.s. of (2.7) that gives the leading contribution, since at least one of the 3 energy differences most involve a higher excitation $\left(\left\langle i\left|\sigma_{l}^{x}\right| j\right\rangle=0\right.$ for $\left.i=j\right)$. For surface spins, $l=1$, (2.7) simplifies:

$$
\chi_{1}^{\mathrm{nl}}=24\left\{\sum_{p, q} \frac{\phi_{p}(1)^{2} \phi_{q}(1)^{2}}{\left(\epsilon_{p}+\epsilon_{q}\right) \epsilon_{p}}\left(\frac{1}{\epsilon_{p}}-\frac{1}{\epsilon_{q}}\right)-\sum_{p}\left(\frac{\phi_{p}(1)}{\epsilon_{p}}\right)^{2} \sum_{q} \frac{\left|\phi_{q}(1)\right|^{2}}{\epsilon_{q}}\right\}
$$

Next we consider the energy-density correlation function at site $l, G_{l}^{e}$, defined by:

$G_{l}^{e}(\tau)=\left\langle 0\left|\sigma_{l}^{z}(\tau) \sigma_{l}^{z}(0)\right| 0\right\rangle-\left\langle 0\left|\sigma_{l}^{z}(\tau)\right| 0\right\rangle\left\langle 0\left|\sigma_{l}^{z}(0)\right| 0\right\rangle$

$$
=\sum_{i>0}\left|\left\langle 0\left|\sigma_{l}^{z}\right| 0\right\rangle\right|^{2} \exp \left[-\tau\left(E_{i}-E_{0}\right)\right]
$$

In the free-fermion representation it is given by: 
$G_{l}^{e}(\tau)=\sum_{\delta>\gamma}\left|\psi_{\delta}(l) \phi_{\gamma}(l)-\psi_{\gamma}(l) \phi_{\delta}(l)\right|^{2} \exp \left[-\tau\left(\epsilon_{\delta}+\epsilon_{\gamma}\right)\right]$,

which can be expressed for surface spins as:

$$
G_{1}^{e}(\tau)=\sum_{\delta>\gamma}\left[\frac{\epsilon_{\delta}-\epsilon_{\gamma}}{h_{1}} \phi_{\delta}(1) \phi_{\gamma}(l)\right]^{2} \exp \left[-\tau\left(\epsilon_{\delta}+\epsilon_{\gamma}\right)\right] .
$$

The spin-spin autocorrelation function, $G_{l}$, which is defined as $G_{l}^{e}$ in (2.9) by replacing $\sigma_{l}^{z}$ by $\sigma_{l}^{x}$, is generally complicated and can be expressed in the form of Pfaffians 21, 14]. Exception is the autocorrelation function for surface spins, which is simply given by:

$$
G_{1}(\tau)=\sum_{q}\left|\Phi_{q}(1)\right|^{2} \exp \left(-\tau \epsilon_{q}\right) .
$$

\section{PHENOMENOLOGICAL AND SCALING CONSIDERATIONS}

As described in the Introduction the Griffiths-McCoy singularities in the paramagnetic phase are connected to the presence of strongly coupled clusters, which are locally in the "ordered phase" and therefore the corresponding excitation energy is very small. For the RTIM the origin of these clusters can be explained either through the analysis of the RG fixed-point distribution [10], which works only in the vicinity of the critical point, or by using simple explicit expressions for the excitation energy 22, 14 and estimate those through random walk arguments 14. Here we use a simple phenomenological approach [1.|6. 23], whose results are in agreement with the above microscopic methods.

Consider the quantity, $P_{L}(N)$, which measures the probability that in a chain of $L$ sites there is a cluster of $N \ll L$ strongly coupled spins. Since $N$ consecutive strong bonds can be found with exponentially small probability $\sim \exp (-A N)$, whereas the cluster could be placed at $\sim L$ different sites we have

$$
P_{L}(N) \sim L \exp (-A N) .
$$

The excitation energy of this sample corresponds to the energy needed to flip all spins in the cluster, which is exponentially small in $N$ :

$$
\epsilon_{1} \sim \exp (-B N) .
$$

Combining (3.1) with (3.2) we have for the probability distribution of the first gap

$$
P_{L}\left(\ln \epsilon_{1}\right) \sim L \epsilon_{1}^{1 / z},
$$

for $\epsilon_{1} \rightarrow 0$ and $1 / z=A / B$. Here, from the scaling combination in eq(3.3): $L \sim \epsilon_{1}^{-1 / z} \sim \tau^{1 / z}$, we can identify $z$ as the dynamical exponent.

Next, we consider the second gap, $\epsilon_{2}$, which is connected to the existence of a second strongly connected cluster of $N^{\prime} \leq N$ spins, and its value corresponds to the energy needed to flip all the spins in the second cluster simultaneously, consequently

$$
\epsilon_{2} \sim \exp \left(-B N^{\prime}\right) .
$$

The probability with which a cluster of size $N^{\prime}$ occurs, provided another cluster of size $N \geq N^{\prime}$ exists, is given by $P_{L}^{\prime}\left(N^{\prime}\right) \sim L \exp \left(-A N^{\prime}\right) \sum_{N=N^{\prime}}^{L} P_{L}(N)$. For $N^{\prime} \ll L$ (or in the infinite system size limit $L \rightarrow \infty$ ) this can be estimated as:

$$
P_{L}^{\prime}\left(N^{\prime}\right) \sim L^{2} \exp \left[-2 A N^{\prime}\right] .
$$

Thus from (3.4) and (3.5) we have

$$
P_{L}^{\prime}\left(\ln \epsilon_{2}\right) \sim L^{2} \epsilon_{2}^{1 / z^{\prime}},
$$

with $1 / z^{\prime}=2 A / B$, thus

$$
z^{\prime}=z / 2 .
$$

Note that the scaling combination in the r.h.s. of (3.6) is dimensionless, as it should be. Repeating the above argument for the third, or generally the $n$th gap the corresponding distribution is described by an exponent $z^{(n)}=z / n$, however the finite size corrections for these gaps are expected to increase rapidly with $n$.

The scaling behavior of the probability distribution of the susceptibilities can be obtained by noticing that both for $\chi_{l}$ and $\chi_{l}^{\mathrm{nl}}$ the leading size dependence is connected with energy gaps in the numerator of (2.4) and (2.7), respectively. Then for the asymptotic behavior of the distribution of the local susceptibility we have:

$$
\ln \left[P\left(\ln \chi_{l}\right)\right]=-\frac{1}{z} \ln \chi_{l}+\text { const, }
$$

like to the inverse gap. For the non-linear susceptibility the second term in the r.h.s. of Eq.(2.7) gives the singular contribution, so that

$$
\ln \left[P\left(\ln \chi_{l}^{\mathrm{nl}}\right)\right]=-\frac{1}{z^{\mathrm{nl}}} \ln \chi_{l}^{\mathrm{nl}}+\text { const },
$$

with

$$
z^{\mathrm{nl}}=3 z
$$

since the asymptotic distribution is the same as that of the third power of the inverse gap. We note that the relation in (3.10) corresponds to the phenomenological result in $[6]$.

The scaling behavior of the average spin autocorrelation function is given by: 


$$
\left[G_{l}\right]_{\mathrm{av}}(\tau)=\int P_{L}\left(\epsilon_{1}\right)\left|M_{l}\right|^{2} \exp \left(-\tau \epsilon_{1}\right) \mathrm{d} \epsilon_{1},
$$

where the factor with the matrix-element is $\left|M_{l}\right|^{2} \sim 1 / L$, since the probability that a low energy cluster is localized at a given site, $l$, is inversely proportional to the length of the chain. Then using (3.3) one arrives to the result in (1.1), thus establishing the relation between the decay exponent of the spin autocorrelation function and the dynamical exponent.

For energy-density autocorrelations, according to (2.10) and (2.11) the characteristic energy scale is $\epsilon_{2}$ and the asymptotic behavior of the average energy-density autocorrelation function is given by:

$$
\left[G_{l}^{e}\right]_{\mathrm{av}}(\tau)=\int P_{L}^{\prime}\left(\epsilon_{2}\right)\left|M_{l}^{e}\right|^{2} \exp \left(-\tau \epsilon_{2}\right) \mathrm{d} \epsilon_{2} .
$$

Now we take the example of the surface autocorrelation function in (2.11) to show that the factor with the matrixelement, $\left|M_{1}^{e}\right|^{2}$, is proportional to $\epsilon_{2}^{2}$. The remaining factor in (2.11) with the first components of the eigenvectors is expected to scale as $1 / L$ due to similar reasons as for the spin autocorrelations, thus $\left|M_{l}^{e}\right|^{2} \sim L^{-1} \epsilon_{2}^{2}$ and together with (3.6) one has $P_{L}^{\prime}\left(\epsilon_{2}\right)\left|M_{l}^{e}\right|^{2} \sim L \epsilon_{2}^{1 / z^{\prime}+1}$. Before evaluating the integral in (3.12) we note that for a fixed $L$ the expression in (3.12) stays valid up to $\tau \sim L^{z}$. Therefore to obtain the $L$ independent asymptotic behavior in $\tau$ we should instead vary $L$, so that according to $(3.6)$ take $L \sim \epsilon_{2}^{-1 /\left(2 z^{\prime}\right)}$ and in this way we stay within the border of validity of (3.12) for any $\tau$. With this modification we arrive to the result in Eq.1.5) where the decay exponent, $\eta_{e}$, is related to the dynamical exponent as

$$
\eta_{e}=2+\frac{1}{z}
$$

where the relation in (3.7) is used. We expect that the factor, $\left|M_{l}^{e}\right|^{2}$, has the same type of scaling behavior for any position $l$, thus the relation in (3.13) stays valid both for bulk and surface spins. We note that the reasoning above (3.13) applies also for the spin autocorrelation function, in which case in (3.11), however there is no explicit $L$ dependence.

In this way we have established a phenomenological, scaling theory which makes a connection between the unconventional exponents in (3.7), (3.10) (3.13) and the dynamical exponent. In the next Section we confront these relations with numerical results.

\section{NUMERICAL RESULTS}

In the numerical calculations we have considered RTIM chains with up to $L=128$ sites and the average is performed over several 10000 realizations, typically we considered 50000 samples. For some cases, where the finite size corrections were strong, we also made runs with $L=256$, but with somewhat less realizations.
We start to present results about the distribution of the first and second gaps. As illustrated in Fig. 1, both for the uniform and the binary distributions, the asymptotic scaling relations for the distribution of the first two gaps in (3.3) and (3.6) are satisfied. From the asymptotic slopes of the distributions in Fig. 1 we have estimated the $1 / z$ and $1 / z^{\prime}$ exponents for the two largest finite systems, $L=64$ and $L=128$, which are presented in Fig. 2 for different points of the Griffiths phase for the uniform distribution. As seen in the Figure the $z$ exponent calculated from the first gap agrees very well with the analytical results in (1.9). For the $z^{\prime}$ exponent, as calculated from the distribution of the second gap the scaling result in (3.7) is also well satisfied, although the finitesize corrections are stronger than for the first gap. For the third gap, due to the even stronger finite size effects, we have not made a detailed investigation. Extrapolated results at $h_{0}=2$ are found to follow the scaling result $z^{(3)}=z / 3$.

Next, we study distribution of the linear and non-linear local susceptibilities at the surface spin. As demonstrated in Fig. 3 both type of distributions satisfy the respective asymptotic relations in (3.8) and (3.9), from which the critical exponents $z$ and $z^{\mathrm{nl}}$ are calculated. The estimates are shown in Fig. 4 at different points of the Griffiths phase. As seen in the Figure the numerical results for the dynamical exponent, $z$, are again in very good agreement with the analytical results in (1.9) and also the exponent of the non-linear susceptibility, $z^{\mathrm{nl}}$, follows fairly well the scaling relation in (3.10).

Finally, we calculate the average energy-density autocorrelation function. As seen in Fig. $5\left[G^{e}\right]_{\text {av }}(\tau)$ displays a linear region in a log-log plot, the size of which is increasing with $L$, but its slope, which is just the decay exponent, $\eta_{e}$, has only a weak $L$ dependence. The slope of the curve and thus the corresponding decay exponent $\eta_{e}$ has a variation with the parameter $h_{0}$, as illustrated in Fig. 6. The estimated $\eta_{e}$ exponents at the critical point, $h_{0}=1$, and in the Griffiths phase are presented in Fig. 7. As seen in this Figure the variation of $\eta_{e}$ is well described by the form $\eta_{e}(\delta)=\eta_{e}(0)+1 / z(\delta)$. This functional form corresponds to the scaling result in Eq.(3.13), if the critical point correlations decay with

$$
\eta_{e}(0)=2
$$

The numerical calculations with $L=128$ give a slightly higher value $\eta_{e}(0) \approx 2.2[13$. However, the finite-size estimates show a slowly decreasing $\eta_{e}(0)$ with increasing system size. Repeating the calculation with $L=256$ we got $\eta_{e}(0) \approx 2.1$. Thus we can conclude that the scaling relation in (3.13) is probably valid and then Eq.(4.1) is the exact value of the decay exponent of the average critical energy-density autocorrelations [24. 


\section{DISCUSSION}

In this paper we have considered the random transverse-field Ising spin chain and studied different consequences of the Griffiths-McCoy singularities in the paramagnetic phase. Our main conclusion is that all singular quantities can be characterized by power-law singularities and the corresponding varying critical exponents can be related to the $z(\delta)$ dynamical exponent and, for energy-density autocorrelations, to the $\eta_{e}(0)$ critical point exponent. Since the exact value of $z(\delta)$ is known in (1.4) and we expect that also the relation in (4.1) is valid, thus we have a complete, analytical description of the Griffiths phase of the RTIM in one dimension.

One interesting feature of our results concerns the distribution of the higher excitations and the value of the corresponding exponent $z^{(n)}=z / n$. Since the decay of dynamical correlations of general, more complex operators are related to $1 / z^{(n)}=n / z$, we obtain a hierarchy of decay exponents which could be simply expressed by those of a few primary operators. This feature is reminiscent to the tower-like structure of anomalous dimensions in two-dimensional conformal models [25]. Our knowledge about the higher excitations can also be used to estimate the correction to scaling contributions.

Much of the reasoning of our phenomenological scaling considerations in Section 3 stay valid for other random quantum systems. Especially the scaling behavior of the higher gaps and the corresponding relation in Eq.(3.7) should be valid even for higher dimensions and the same is true for the distribution of the non-linear susceptibility and the corresponding relation in (3.10.

In one dimensions the universality class of the RTIM involves several random systems, among others the random quantum Potts chain [26]. For these models one does not expect a universality of the $z(\delta)$ exponent in the Griffiths phase, although scaling relations, like the one in (3.7) are very probably valid. It would be interesting to perform a numerical study on the random quantum Potts model to check the existing conjectures.

Another possible field where the present results could be applied is the problem of anomalous diffusion in a random environment [17, 18,27]. Making use of the exact correspondence [17,18 between the Hamilton operator in (1.2) and the Fokker-Planck operator of the onedimensional random walk we can use the relation in (3.7) to describe the distribution of the eigenvalues of the corresponding Fokker-Planck operator. One can also define analogous quantity to the energy-density autocorrelation function in (2.10) by considering connected persistence correlations, whose asymptotic decay is related to the distribution of the second gap, like in (3.12). Research in this field is in progress.

Acknowledgment: This study has been partially performed during our visits in Köln and Budapest, respectively. F. I.'s work has been supported by the Hungarian National Research Fund under grant No OTKA
TO23642 and by the Ministery of Education under grant No. FKFP 0765/1997. H. R. was supported by the Deutsche Forschungsgemeinschaft (DFG).

[1] See H. Rieger and A. P Young, in Complex Behavior of Glassy Systems, ed. M. Rubi and C. Perez-Vicente, Lecture Notes in Physics 492, p. 256, Springer-Verlag, Heidelberg, 1997.

[2] R.B. Griffiths, Phys. Rev. Lett. 23, 17 (1969).

[3] In a classical random system the singularities in the corresponding Griffiths-region are weaker, e.g. the spin-spin autocorrelation function has an enhanced power law decay (i.e. $\sim \exp \left(-c(\ln t)^{\alpha}\right)$ with $\left.\alpha>1\right)$, in contrast to the power-law dependence observed in quantum systems.

[4] B. McCoy, Phys. Rev. Lett. 23, 383 (1969).

[5] H. Rieger and N. Kawashima, Europ. Phys. J. B, in press (1998); C. Pich, A. P. Young, H. Rieger, and N. Kawashima, Phys. Rev. Lett. 81, in press (1998); T. Ikegami, S. Miyashita and H. Rieger, J. Phys. Soc. Jap. 67, 2761 (1998).

[6] H. Rieger and A.P. Young, Phys. Rev. Lett. 72, 4141 (1994); M. Guo, R.N. Bhatt and D. Huse, Phys. Rev. Lett. 72, 4137 (1994).

[7] W. Wu, B. Ellman, T.F. Rosenbaum, G. Aeppli and D.H. Reich, Phys. Rev. Lett. 67, 2076 (1991); W. Wu, D. Bitko, T.F. Rosenbaum and G. Aeppli, Phys. Rev. Lett. 71, 1919 (1993).

[8] B.M. McCoy and T.T. Wu, Phys. Rev. 176, 631 (1968); 188, 982(1969); B.M. McCoy, Phys. Rev. 188, 1014 (1969).

[9] R. Shankar and G. Murthy, Phys. Rev. B 36, 536 (1987).

[10] D.S. Fisher, Phys. Rev. Lett. 69, 534 (1992); Phys. Rev. B 51, 6411 (1995).

[11] A. P. Young and H. Rieger, Phys. Rev. B 53, 8486 (1996).

[12] F. Iglói and H. Rieger, Phys. Rev. Lett. 78, 2473 (1997).

[13] H. Rieger and F. Iglói, Europhys. Lett. 39, 135 (1997).

[14] F. Iglói and H. Rieger, Phys. Rev. B 57, 11404 (1998).

[15] D.S. Fisher and A.P. Young, Phys. Rev. B 58, 9131 (1998).

[16] A. P. Young, Phys. Rev. B 56, 11691 (1997).

[17] F. Iglói and H. Rieger, Phys. Rev. E 58, 4238 (1998).

[18] F. Iglói, L. Turban and H. Rieger, Phys. Rev. E 59, *** (1999).

[19] E. Lieb, T. Schultz and D. Mattis, Ann. Phys. (N.Y.) 16, 407 (1961); S. Katsura, Phys. Rev. 127, 1508 (1962); P. Pfeuty, Ann. Phys. (Paris) 57, 79 (1970).

[20] F. Iglói and L. Turban, Phys. Rev. Lett. 77, 1206 (1996).

[21] J. Stolze, A. Nöppert and G. Müller, Phys. Rev. B 52, 4319 (1995).

[22] F. Iglói, L. Turban, D. Karevski and F. Szalma, Phys. Rev. B 56, 11031 (1997).

[23] M. J. Thill and D. A. Huse, Physica A, 15, 321 (1995).

[24] Numerical estimates for the decay exponent of the average energy-density autocorrelation function for surface spins at the critical point are $\eta_{e}^{s} \approx 2.5$ with $L=128$ [13], 
which is somewhat larger than for bulk autocorrelations. Discrepancies between estimates for $z$ from surface and bulk quantities have been observed before 14. They can be attributed to corrections to scaling effects which are different for different quantities, see also fig.6 in [16].

[25] J.L. Cardy, in Phase Transitions and Critical Phenomena, vol. 11 eds C. Domb and J.L. Lebowitz (New York: Academic) (1987).

[26] T. Senthil and S. Majumdar, Phys. Rev. Lett. 76, 3001 (1996).

[27] A. Comtet and D. Dean, J. Phys. A 31, 8595 (1998); D. S. Fisher, P. Le Doussal, and C. Monthus, Phys. Rev. Lett. 80, 3539 (1998), cond-mat/9811300.
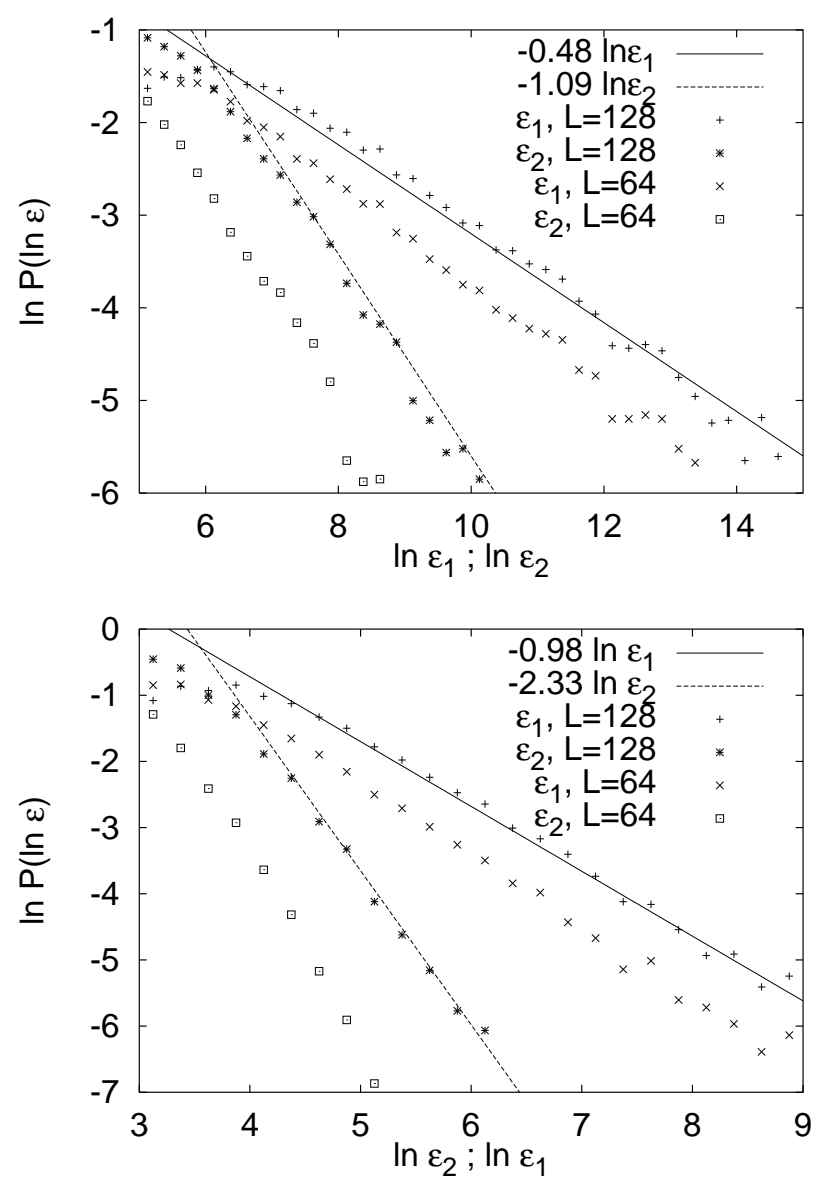

FIG. 1. Probability distribution of $\ln \varepsilon_{1}$ and $\ln \varepsilon_{2}$ for the uniform distribution at $h_{0}=2$ (top) and the binary distribution $(\lambda=4)$ at $h_{0}=2.5$ (bottom). The straight lines are least square fits to the data for the largest system size, their slopes correspond to $1 / z\left(h_{0}\right)$ and $1 / z^{\prime}\left(h_{0}\right)$, respectively. They follow the predicted relation $z^{\prime}\left(h_{0}\right)=z\left(h_{0}\right) / 2$.

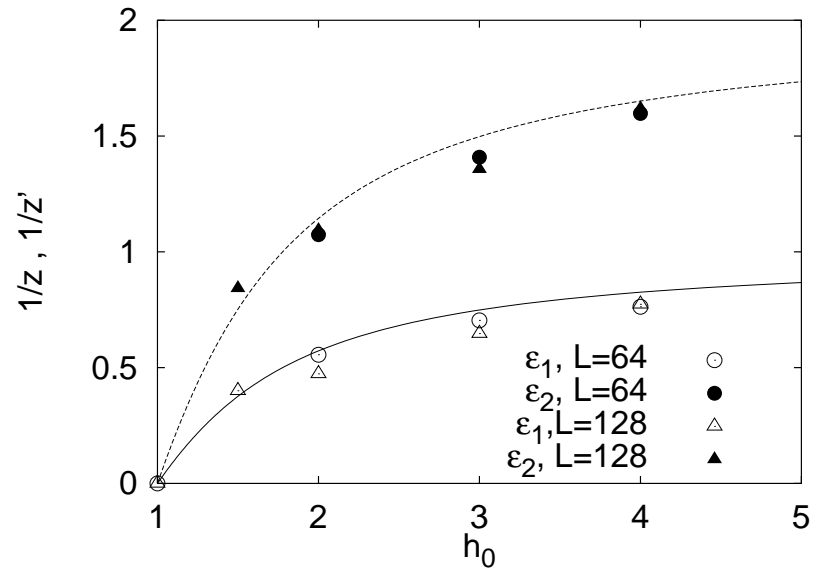

FIG. 2. The estimates for $1 / z$ and $1 / z^{\prime}$ as a function of $h_{0}$ for the uniform distribution. They have been obtained from our analysis of the probability distribution of $\ln \varepsilon_{1}$ and $\ln \varepsilon_{2}$, respectively, for two system sizes (as exemplified in fig. 1). The full line for $1 / z$ corresponds to the analytical result (1.4), the broken line corresponds to $2 / z$, which we predict to be identical with $1 / z^{\prime}$.

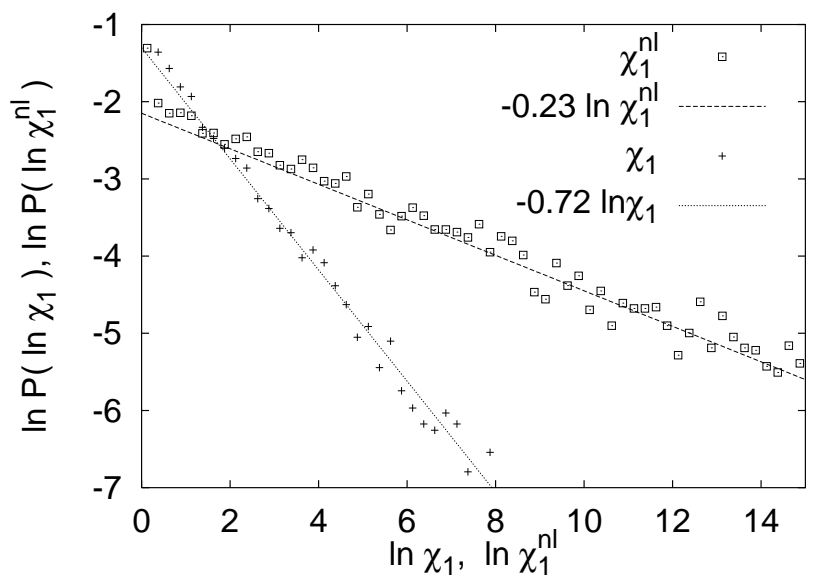

FIG. 3. Probability distribution of the linear and non-linear susceptibility, $\ln \chi_{1}$ and $\ln \chi_{1}^{\mathrm{nl}}$, respectively, for the uniform distribution at $h_{0}=3$. The straight lines are least square fits to the data for the largest system size, their slopes correspond to $1 / z\left(h_{0}\right)$ and $1 / z^{\mathrm{nl}}\left(h_{0}\right)$, respectively. They follow the predicted relation $z^{\mathrm{nl}}\left(h_{0}\right)=3 z\left(h_{0}\right)$. 


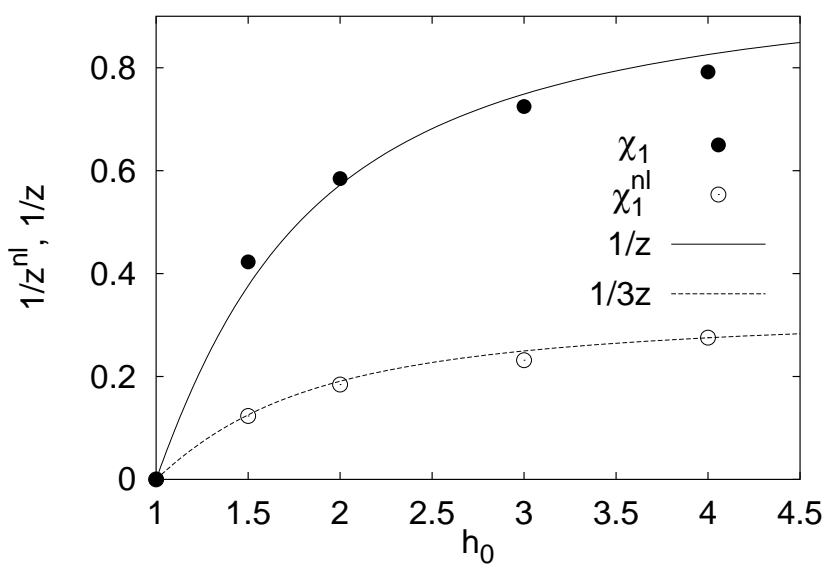

FIG. 4. The estimates for $1 / z$ and $1 / z^{\mathrm{nl}}$ as a function of $h_{0}$ for the uniform distribution. They have been obtained from our analysis of the probability distribution of $\ln \chi_{1}$ and $\ln \chi_{1}^{\mathrm{nl}}$, respectively, for two system sizes (as exemplified in fig. 3). The full line for $1 / z$ corresponds to the analytical result (1.4), the broken line corresponds to $1 / 3 z$, which should be identical with $1 / z^{\text {nl }}$.

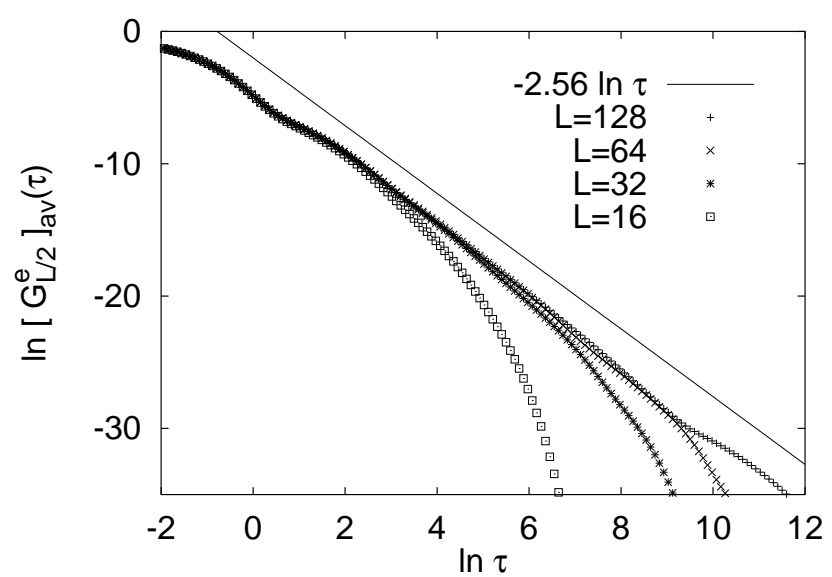

FIG. 5. The bulk energy-energy autocorrelation function $\left[G_{L / 2}^{e}\right]_{\mathrm{av}}(\tau)$ for the binary distribution $(\lambda=4)$ at $h_{0}=1.5$ for different system sizes as a function of $\ln \tau$. The slope of the straight line identifies the exponent $\eta_{e}\left(h_{0}\right)$ describing the asymptotic decay of $\left[G_{L / 2}^{e}\right]_{\mathrm{av}}(\tau)$ in the infinite system size limit $L \rightarrow \infty$.

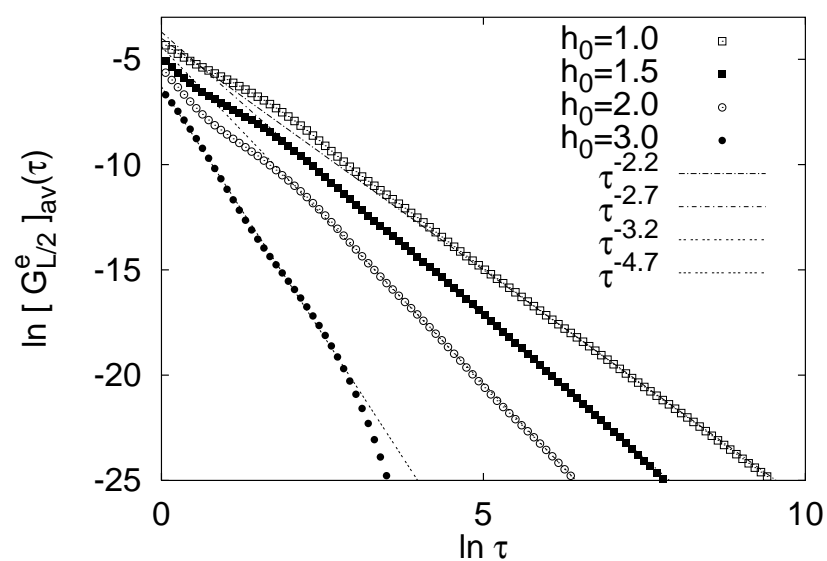

FIG. 6. The bulk energy-energy autocorrelation function $\left[G_{L / 2}^{e}\right]_{\text {av }}(\tau)$ for the binary distribution $(\lambda=4)$ at different values for $h_{0}$ for $L=128$ as a function of $\ln \tau$. One observes the variation of the exponent $\eta_{e}\left(h_{0}\right)$ (identical to the slope of the straight line fits) with increasing $h_{0}$.

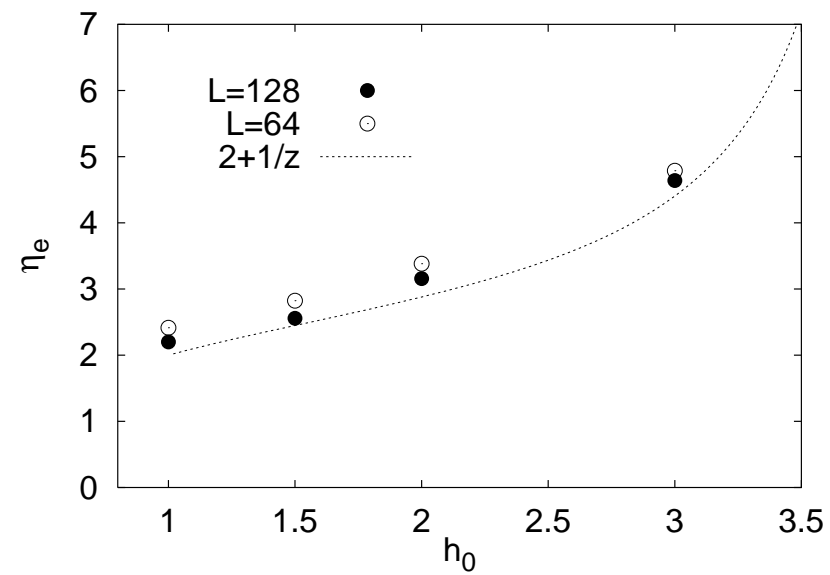

FIG. 7. The exponent $\eta_{e}\left(h_{0}\right)$ for the binary distribution $(\lambda=4)$ as obtained from the analysis of the asymptotic decay of the bulk energy-energy autocorrelation function $\left[G_{L / 2}^{e}\right]_{\mathrm{av}}(\tau)$ á la fig. 6 . The full line is the analytical prediction $\eta_{e}\left(h_{0}\right)=2+1 / z\left(h_{0}\right)$ with $z\left(h_{0}\right)$ given by the exact formula 1.4 for the binary distribution with $\lambda=4$. 